\title{
Static output-feedback control under information structure constraints ${ }^{\underline{t}}$
}

\author{
J. Rubió-Massegúa, ${ }^{\mathrm{a}, *}$ J. M. Rossell ${ }^{\mathrm{a}}$, H. R. Karimi ${ }^{\mathrm{b}}$, F. Palacios-Quiñonero ${ }^{\mathrm{a}}$ \\ ${ }^{a}$ Department of Applied Mathematics III, Universitat Politècnica de Catalunya (UPC), 08242-Manresa, Barcelona, Spain \\ ${ }^{b}$ Department of Engineering, University of Agder, Faculty of Engineering and Science, N-4898 Grimstad, Norway
}

\begin{abstract}
An important challenge in the static output-feedback control context is to provide an isolated gain matrix possessing a zero-nonzero structure, mainly in problems presenting information structure constraints. Although some previous works have contributed some relevant results to this issue, a fully satisfactory solution has not yet been achieved up to now. In this note, by using a Linear Matrix Inequality approach and based on previous results given in the literature, we present an efficient methodology which permits to obtain an isolated static output-feedback gain matrix having, simultaneously, a zero-nonzero structure imposed a priori.
\end{abstract}

Keywords: Information structure constraints, output-feedback control, LMIs.

\section{Introduction}

Nowadays, robust state-feedback controllers for a wide class of systems containing disturbances, nonlinearities, uncertainties and delays are usually obtained by solving convex optimization problems with Linear Matrix Inequality (LMI) constraints (Boyd, Ghaoui, Feron, and Balakrishnan, 1994). Moreover, for problems involving a structured set of subsystems with restricted information exchange, this LMI approach also allows to impose special zero-nonzero structures on the LMI variables in order to produce a control matrix whose structure is in accordance with the information structure constraints of the system.

A serious drawback in practical implementation of statefeedback controllers is that a full knowledge of the state vector is rarely available. In the static output-feedback approach, the goal is to find a controller that computes the vector of control actions directly from the vector of observed variables, which usually are linear combinations of the states. The main difficulty to attain this goal is that, following the usual LMI approach, an implicit relation results and, in general, the outputfeedback gain matrix, namely $K$, cannot be directly isolated. A relevant contribution to this problem can be found in the works by Zečević and Šiljak (2004, 2008, 2010), where several explicit expressions for $K$ are provided. However, in order to obtain a zero-nonzero structure, it would be desirable to have a simpler expression for $K$, since this would help to make the structural constraints on the LMI variables less restrictive and, therefore, to supply more degrees of freedom to achieve a proper solution of the LMIs involved in the process.

\footnotetext{
This work was supported in part by the Spanish Ministry of Economy and Competitiveness under Grant DPI2011-25567-C02 and by the Norwegian Center of Offshore Wind Energy (NORCOWE) under Grant 193821/S60 from the Research Council of Norway (RCN).

${ }^{*}$ Corresponding author. Tel.: +34 93 8777251, fax: +34938777202

Email addresses: josep.rubio@upc.edu (J. Rubió-Massegú),

josep.maria.rossell@upc.edu (J. M. Rossell),

hamid.r.karimi@uia.no (H. R. Karimi),

francisco.palacios@upc.edu (F. Palacios-Quiñonero)
}

In the present paper, based on some simplifications of the gain matrices used in the previous works, we obtain an effective LMI formulation to design static output-feedback controllers similar to the existing one for the state-feedback problem. Moreover, by exploiting this similarity, a strategy to obtain structured output gain matrices is provided. Due to its generality, conceptual simplicity and ease of implementation, the proposed strategy can be a significant tool to be considered in a wide variety of control problems involving a partial knowledge of the state variables and constrained information exchange.

The rest of the paper is organized as follows. Section 2 presents the problem statement. In Section 3, the matrices used in Zečević and Šiljak $(2004,2008,2010)$ are transformed in such a way that the output gain matrix takes a similar form to that of the state gain matrix. Section 4 is devoted to developing a strategy to compute the output gain matrices. In Section 5, an academic numerical example to illustrate the effectiveness of the proposed methodology is presented and, finally, conclusions are drown in Section 6.

\section{Problem statement}

Consider an LMI which depends on a symmetric positivedefinite $n \times n$ matrix $X$, an $m \times n$ matrix $Y$, and possibly other matrix variables or scalars. Since the LMI may contain both positive-definite and positive semi-definite terms, we write it in a compact form as

$$
F(X, Y, z)>0, \quad G(X, Y, z) \geqslant 0, \quad X>0,
$$

where $z$ is the vector containing the scalars and the entries of the matrix variables distinct from $X$ and $Y$. This kind of LMI appears in a large amount of control problems in the context of static state-feedback on both discrete and continuous time, where the state gain matrix, namely $\tilde{K}$, is explicitly given by $\tilde{K}=Y X^{-1}$ (see Boyd et al. (1994), or the more recent works by Amato et al. (2010); Chen and Wang (2012); Dhawan and Kar (2011); Du et al. (2011); Huang and Mao (2009); Liu et al. 
(2011); Oishi and Fujioka (2010); Wang et al. (2010); Wang and Shen (2011) and references therein).

In static output-feedback control problems, the state gain matrix factors as the product $\tilde{K}=K C$, where $C$ is a given $q \times n$ full row-rank matrix with $q<n$, and $K$ is the output gain matrix with dimensions $m \times q$. Consequently, when an output gain matrix $K$ is required, the following problem arises:

Problem 1. Find a solution of the LMI given in (1) such that the product $Y X^{-1}$ factors as

$$
Y X^{-1}=K C
$$

where $K$ is a matrix with dimensions $m \times q$.

To solve this problem, Zečević and Šiljak (2004, 2008, 2010) considered matrices of the form

$$
\begin{aligned}
& X=\rho X_{0}+Q X_{Q} Q^{T}+U X_{C} U^{T}, \\
& Y=Y_{C} U^{T},
\end{aligned}
$$

where $\rho>0$ is an LMI variable, $X_{Q}, X_{C}, Y_{C}$ are matrix variables with $X_{Q}, X_{C}$ symmetric, and $X_{0}, Q, U$ are constant matrices satisfying $X_{0} C^{T}=U, C Q=0$, with $X_{0}$ symmetric. The authors proved that if $X$ and $\rho X_{0}+Q X_{Q} Q^{T}$ are nonsingular matrices, then $Y X^{-1}=K C$ with

$$
K=\rho^{-1} Y_{C}\left[I-U^{T} S R\right],
$$

where $I$ is an identity matrix, and

$$
S=\left[\rho X_{0}+Q X_{Q} Q^{T}\right]^{-1} U X_{C}, \quad R=\left[I+U^{T} S\right]^{-1} .
$$

Observe that the expression for $K$ given in (4), although explicit, is so intricate that imposing additional conditions on this matrix, as a given zero-nonzero structure, might demand severe structural constraints on the matrix variables $X_{Q}, X_{C}, Y_{C}$, which might lead to poor solutions of the LMI (1) or even infeasibility.

In this paper, by means of a convenient change of the matrix variables in (3), we simplify equations (4) and (5) so that $K$ takes the form $K=Y_{R} X_{R}^{-1}$, where $Y_{R}, X_{R}$ are new matrix variables. This simple expression for $K$ is analogous to that obtained for the state gain matrix $\tilde{K}=Y X^{-1}$, consequently the usual strategy used to produce structured state gain matrices can be employed to obtain structured output gain matrices. This will result in a conceptually simple and easy-to-implement method for output-feedback controller design under information structure constraints.

\section{Preliminary results}

The purpose of this section is to express the matrices $X, Y$ in (3) in a more appropriate way. In the sequel, $Q$ is an $n \times(n-q)$ matrix such that its columns are a basis of $\operatorname{Ker}(C), X_{0}$ is a given symmetric $n \times n$ matrix and $U=X_{0} C^{T}$.

Since $C U=C X_{0} C^{T}, C U$ is symmetric. Moreover, for the LMI (1) to be feasible with matrices of the form (3), it is necessary to assume that $C U$ is invertible. To prove this, let $X$ be as in (3) and suppose that $X>0$. From $C Q=0$ and $C X_{0} C^{T}=C U$, it follows that

$$
0<C X C^{T}=\rho C U+C U X_{C} U^{T} C^{T}=C U\left[\rho I+X_{C} U^{T} C^{T}\right],
$$

and, consequently, $C U$ is invertible.
Theorem 1. The matrices $X, Y$ given in (3) can be written in the form $X=Q \tilde{X}_{Q} Q^{T}+R \tilde{X}_{R} R^{T}, Y=\tilde{Y}_{R} R^{T}$, where $\tilde{X}_{Q}, \tilde{X}_{R}, \tilde{Y}_{R}$ are matrices of appropriate dimensions with $\tilde{X}_{Q}, \tilde{X}_{R}$ symmetric, and $R$ is given by $R=U(C U)^{-1}$.

We can observe that matrix $R$ in the statement satisfies the matrix identity $C R=I$. To prove Theorem 1 , first we need a technical lemma which explicitly solves the homogeneous equation $C \bar{X}=0$ with $\bar{X}$ symmetric.

Lemma 1. For a given $n \times n$ symmetric matrix $\bar{X}$, the following two conditions are equivalent: (a) $C \bar{X}=0$, (b) $\bar{X}=Q X_{Q} Q^{T}$, where $X_{Q}$ is a symmetric matrix. Moreover, if $(a)$ and $(b)$ are satisfied, then (b) holds with $X_{Q}=Q^{\dagger} \bar{X}\left(Q^{\dagger}\right)^{T}$, where $Q^{\dagger}$ is the Moore-Penrose pseudoinverse of $Q$, i.e. $Q^{\dagger}=\left(Q^{T} Q\right)^{-1} Q^{T}$.

PROOF. Clearly (b) implies (a). Suppose (a) holds and let $X_{Q}$ be the matrix $X_{Q}=Q^{\dagger} \bar{X}\left(Q^{\dagger}\right)^{T}$. From (a), we have $\bar{X}=Q S$ for an $(n-q) \times n$ matrix $S$. Then, $Q Q^{\dagger} \bar{X}=Q Q^{\dagger} Q S=Q S=\bar{X}$ and $Q X_{Q} Q^{T}=Q Q^{\dagger} \bar{X}\left(Q^{\dagger}\right)^{T} Q^{T}=\bar{X}\left(Q^{\dagger}\right)^{T} Q^{T}=\left(Q Q^{\dagger} \bar{X}\right)^{T}=\bar{X}^{T}=$ $\bar{X}$, concluding the proof of Lemma 1 .

PROOF (Proof of Theorem 1). Consider the matrices

$$
\begin{aligned}
& \tilde{X}_{C}=C U X_{C} C U+\rho C U, \\
& \tilde{X}_{Q}=X_{Q}+\rho M_{0}, \\
& \tilde{Y}_{C}=Y_{C} C U,
\end{aligned}
$$

where $M_{0}$ is given by $M_{0}=Q^{\dagger}\left[X_{0}-U(C U)^{-1} U^{T}\right]\left(Q^{\dagger}\right)^{T}$. Taking into account that $X_{0} C^{T}=U$, which implies $C X_{0}=U^{T}$, we have $C\left[X_{0}-U(C U)^{-1} U^{T}\right]=0$. By Lemma 1, we get

$$
Q M_{0} Q^{T}=X_{0}-U(C U)^{-1} U^{T}
$$

From (3), (7) and (8), and after some elementary manipulations, it is easy to see that

$$
\begin{aligned}
& X=Q \tilde{X}_{Q} Q^{T}+U(C U)^{-1} \tilde{X}_{C}(C U)^{-1} U^{T}, \\
& Y=\tilde{Y}_{C}(C U)^{-1} U^{T}
\end{aligned}
$$

which is the form given in the statement with $\tilde{X}_{R}=\tilde{X}_{C}, \tilde{Y}_{R}=\tilde{Y}_{C}$ and $R=U(C U)^{-1}$. This completes the proof.

\section{Output-feedback controller design}

In this section, a useful methodology to get an isolated outputfeedback control gain matrix possessing, simultaneously, a preassigned zero-nonzero structure, is developed.

Denote $X_{Q}=\tilde{X}_{Q}, X_{R}=\tilde{X}_{R}$, and $Y_{R}=\tilde{Y}_{R}$ in Theorem 1 . Then, we have

$$
\begin{aligned}
& X=Q X_{Q} Q^{T}+R X_{R} R^{T}, \\
& Y=Y_{R} R^{T},
\end{aligned}
$$

where $X_{Q}$ and $X_{R}$ are $(n-q) \times(n-q)$ and $q \times q$ symmetric matrices, respectively, and $Y_{R}$ is an $m \times q$ matrix. In (10), $Q$ is an $n \times(n-q)$ matrix whose columns are a basis of $\operatorname{Ker}(C)$, and $R$ is a given $n \times q$ matrix such that $C R=I$ ( $R$ is a right inverse of $C$ ). Notice that $R$ can be parameterized as

$$
R=C^{\dagger}+Q L,
$$


where $L$ is an $(n-q) \times q$ matrix and $C^{\dagger}$ is the Moore-Penrose pseudoinverse of $C$, which is explicitly given by $C^{\dagger}=C^{T}\left(C C^{T}\right)^{-1}$. Indeed, $C\left(C^{\dagger}+Q L\right)=I$, and if $C R=I$ then $C\left(R-C^{\dagger}\right)=0$. Hence, $R=C^{\dagger}+Q L$ for an $(n-q) \times q$ matrix $L$.

In terms of the new matrix variables, the gain matrix obtained from expressions (4) and (5) takes a very simple form, as shown in the following theorem.

Theorem 2. Suppose that $X$ in (10) is nonsingular. Then, $X_{R}$ is nonsingular and $Y X^{-1}=K C$ with $K=Y_{R} X_{R}^{-1}$.

PROOF. Pre-multiplying the first equation in (10) by $C$, and taking into account that $C Q=0$ and $C R=I$, it follows that $C X=X_{R} R^{T}$. Since $X$ is nonsingular, $C X$ has rank $q$. Therefore, $X_{R}$ has rank $q$ and $X_{R}$ is nonsingular since $X_{R}$ is a $q \times q$ square matrix. Moreover,

$$
Y=Y_{R} R^{T}=Y_{R} X_{R}^{-1} X_{R} R^{T}=Y_{R} X_{R}^{-1} C X .
$$

Post-multiplying both sides of (12) by $X^{-1}$, we get $Y X^{-1}=K C$ with $K=Y_{R} X_{R}^{-1}$, and the proof is concluded.

We can now summarize the controller design in the following steps:

Step 1. Choose an $(n-q) \times q$ matrix $L$ and compute $R=C^{\dagger}+Q L$.

Step 2. Substitute the matrices $X, Y$ given in (10) into the LMI (1). The new LMI has $X_{Q}, X_{R}, Y_{R}$, and $z$ as variables.

Step 3. Find a solution $\left(X_{Q}, X_{R}, Y_{R}, z\right)$ of this LMI, when it is feasible, and compute the matrices $X, Y$ given in (10). Then, the triplet $(X, Y, z)$ is a solution of (1) satisfying $Y X^{-1}=K C$, where $K=Y_{R} X_{R}^{-1}$.

Observe that the expression obtained for the gain matrix $K=Y_{R} X_{R}^{-1}$ allows to impose a preassigned structure on $K$ by taking $X_{R}$ and $Y_{R}$ in the usual way, that is, $X_{R}$ as a blockdiagonal matrix and $Y_{R}$ with the zero-nonzero structure desired for $K$. For example, if we are interested in obtaining a gain matrix of the form

$$
K=\left[\begin{array}{ccc}
K_{11} & K_{12} & 0 \\
0 & 0 & K_{23} \\
K_{31} & 0 & K_{33}
\end{array}\right]
$$

then we take

$$
X_{R}=\left[\begin{array}{ccc}
X_{1} & 0 & 0 \\
0 & X_{2} & 0 \\
0 & 0 & X_{3}
\end{array}\right], \quad Y_{R}=\left[\begin{array}{ccc}
Y_{11} & Y_{12} & 0 \\
0 & 0 & Y_{23} \\
Y_{31} & 0 & Y_{33}
\end{array}\right],
$$

in order to achieve the desired structure on the gain matrix $K$. It is worth noting that $X_{Q}$ is a full matrix, and that the blocks of $X_{R}$ and $Y_{R}$ might contain a very large number of free entries, specially in large-scale systems.

Remark 1. (i) It should be noted that following this strategy, the number of free LMI variables is greater than or equal to the number of free LMI variables that are obtained using the strategies proposed in Zečević and Šiljak (2010), which are based on employing the expressions (3), (4) and (5), while constraining the values of the matrices $X_{Q}, X_{C}, Y_{C}$. (ii) The proposed strategy depends on the choice of $L$, which plays a similar role to the matrix $X_{0}$ in (3) but with smaller dimensions. This has the advantage that less parameters have to be previously chosen.

(iii) For the problem of stabilizing a continuous linear timeinvariant system with a nonlinear term, some heuristic choices for $L$ can be deduced from the (heuristic) choices for $X_{0}$ in Zečević and Siljak (2010) and the relationship between $L$ and $X_{0}$, that can be easily obtained from (11) (which yields $L=Q^{\dagger} R$ ) and the equations $R=U(C U)^{-1}$, $U=X_{0} C^{T}$

In view of Remark 1, the proposed methodology appears to be suitable for large-scale systems. The next proposition allows us to reduce the dimensionality of the term $X=Q X_{Q} Q^{T}+$ $R X_{R} R^{T}>0$, which appears in the LMI of Step 2 .

Proposition 1. The matrix inequality $\left[Q X_{Q} Q^{T}+R X_{R} R^{T}\right]>0$ is equivalent to $X_{Q}>0$ and $X_{R}>0$.

PROOF. We have

$$
Q X_{Q} Q^{T}+R X_{R} R^{T}=\left[\begin{array}{ll}
Q & R
\end{array}\right]\left[\begin{array}{cc}
X_{Q} & 0 \\
0 & X_{R}
\end{array}\right]\left[\begin{array}{ll}
Q & R
\end{array}\right]^{T} .
$$

The $n \times n$ matrix $P=\left[\begin{array}{ll}Q R & R\end{array}\right]$ is nonsingular, because if $Q u+R v=$ 0 , where $u$ and $v$ are vectors of appropriate dimensions, then $0=C[Q u+R v]=C R v=v$ and, therefore, $v=0$. This implies that $Q u=0$, hence $u=v=0$. Now, from (15) we have that $\left[Q X_{Q} Q^{T}+R X_{R} R^{T}\right]>0$ if and only if $\operatorname{diag}\left[X_{Q}, X_{R}\right]>0$, which is equivalent to $X_{Q}>0$ and $X_{R}>0$.

To end this section, we deal with optimization problems of the form

$$
\left\{\begin{array}{l}
\text { Minimize } h(X, Y, z) \\
\text { subject to the LMI (1) and the constraint (2) }
\end{array}\right.
$$

where the objective function $h$ is assumed to be linear. We can associate to this non-convex problem an LMI optimization problem:

$$
\left\{\begin{array}{l}
\text { Minimize } h(X, Y, z) \\
\text { subject to the LMI (1), } X, Y \text { as in (10) }
\end{array}\right.
$$

where the optimization variables are $X_{Q}, X_{R}, Y_{R}$ and $z$. Clearly, an optimal solution of the convex problem (17) is not necessarily an optimal solution of (16), but it minimizes the objective function on a set that satisfies all the constraints in (16). In particular, the constraint (2) is satisfied with $K=Y_{R} X_{R}^{-1}$. Obviously, solving (17) with an appropriate structure on the matrices $X_{R}, Y_{R}$, a preassigned zero-nonzero structure on the gain matrix $K$ can also be achieved.

\section{Numerical example}

In this section, the effectiveness of the proposed methodology is illustrated by means of an academic example. A practical application of the theoretical results presented in this paper in the field of Structural Vibration Control can be found in RubióMassegú et al. (2012). 


\subsection{An $H_{\infty}$ control approach}

The standard $H_{\infty}$ control procedure for continuous linear time-invariant systems with static state-feedback control involves solution of the following LMI (Boyd et al., 1994):

$$
\left[\begin{array}{cc}
A X+X A^{T}+B Y+Y^{T} B^{T}+\gamma^{-2} B_{w} B_{w}^{T} & * \\
C_{z} X+D_{z} Y & -I
\end{array}\right]<0,
$$

where the unknowns $X>0, Y$, and the constant matrices $A, B$, $B_{w}, C_{z}, D_{z}$, are of appropriate dimensions, $\gamma>0$ is a scalar parameter which bounds the $H_{\infty}$-norm of the system (induced $L^{2}$ gain), and (*) denotes the symmetric entry. The corresponding state-feedback gain matrix is then given by $\tilde{K}=Y X^{-1}$. Introducing the parameter $\eta=\gamma^{-2}$, the smallest value of $\gamma$ under static output-feedback can be achieved by solving the following optimization problem:

$$
\left\{\begin{array}{l}
\text { Maximize } \eta \\
\text { subject to } X>0, \eta>0, \text { LMI (18) and constraint (2) }
\end{array}\right.
$$

which has the form given in (16). The matrix values are

$$
\begin{aligned}
A & =\left[\begin{array}{rrrrr}
-4 & 0 & -2 & 0 & 0 \\
0 & -2 & 0 & 2 & 0 \\
0 & 0 & -2 & 0 & -1 \\
0 & -2 & 0 & -1 & 0 \\
3 & 0 & -2 & 0 & -1
\end{array}\right], & B=\left[\begin{array}{lll}
1 & 0 & 0 \\
1 & 0 & 0 \\
0 & 0 & 0 \\
0 & 1 & 0 \\
0 & 0 & 1
\end{array}\right], & B_{w}=\left[\begin{array}{l}
1 \\
1 \\
1 \\
1 \\
1
\end{array}\right], \\
C & =\left[\begin{array}{rrrrr}
1 & 0 & 0 & 0 & 0 \\
0 & 1 & 0 & 0 & 0 \\
0 & 0 & 0 & 0 & 1
\end{array}\right], & C_{z}=\left[\begin{array}{c}
I_{5} \\
0 \\
03 \times 5
\end{array}\right], & D_{z}=\left[\begin{array}{c}
0_{5 \times 3} \\
I_{3}
\end{array}\right] .
\end{aligned}
$$

We can observe that $A$ is unstable. Matrix $Q$ is selected as $Q=\left[\begin{array}{lllll}0 & 0 & 1 & 0 & 0 \\ 0 & 0 & 0 & 1 & 0\end{array}\right]^{T}$ and matrix $L$ is chosen as $L=[0]_{2 \times 3}$. Then, matrix $R$ given in (11) is computed as

$$
R=\left[\begin{array}{lll}
1 & 0 & 0 \\
0 & 1 & 0 \\
0 & 0 & 0 \\
0 & 0 & 0 \\
0 & 0 & 1
\end{array}\right]
$$

In this example three cases are presented, which depend on the preassigned zero-nonzero structure of the gain matrix $K$ : (a) a full output-feedback gain matrix; (b) a tridiagonal gain matrix; and (c) a diagonal gain matrix (totally decentralized control). In each case, the $\gamma$-value is calculated.

Case (a). In this first case, no special structure is imposed on the matrix $K$. According to the strategy presented in Section 4 , the computed gain matrix is given by

$$
K=\left[\begin{array}{rrr}
-3.9461 & -0.9267 & -0.9166 \\
-2.9743 & -0.0871 & 0.7084 \\
-1.2371 & 0.3184 & -2.5569
\end{array}\right]
$$

In this case, we get $\gamma_{a}=1.2084$.

Case (b). Suppose that we are interested in obtaining a tridiagonal gain matrix $K$. Then, we impose a diagonal structure on the matrix $\left[X_{R}\right]_{3 \times 3}$ and a tridiagonal form on the matrix $\left[Y_{R}\right]_{3 \times 3}$ in order to get the same structure in the gain matrix $K$. In this case, we have

$$
K=\left[\begin{array}{ccc}
-7.8313 & -0.5934 & 0 \\
-2.5153 & -0.1913 & -0.0158 \\
0 & 0.0023 & -2.9050
\end{array}\right]
$$

with a minimum $\gamma$-value of $\gamma_{b}=1.2385$.

Case (c). To achieve a diagonal structure in the matrix $K$, we impose a diagonal structure on the matrices $\left[X_{R}\right]_{3 \times 3}$ and $\left[Y_{R}\right]_{3 \times 3}$. In this case, we obtain

$$
K=\left[\begin{array}{ccc}
-6.4172 & 0 & 0 \\
0 & 0.0458 & 0 \\
0 & 0 & -3.9087
\end{array}\right]
$$

with $\gamma_{c}=1.2859$

It is worth noting that $\gamma_{a}<\gamma_{b}<\gamma_{c}$, since the number of variables increases when less structural conditions are imposed on the matrix variables involved in the LMI and, consequently, it has more degrees of freedom to attain a better (minimum) $\gamma$-value.

\section{Conclusions}

In this paper, a Linear Matrix Inequality strategy to design static output-feedback controllers has been presented. The most remarkable feature of the proposed solution is its simplicity, which makes it possible to design semi-decentralized static output-feedback controllers in a similar way to semi-decentralized static state-feedback controllers. The question of finding a proper choice of matrix $L$ remains open, which is an interesting challenge for future research. From a practical point of view, the proposed methodology can be of great interest to a wide variety of engineering areas, where complex control problems involving several subsystems, with constrained information exchange, and a partial knowledge of the state variables, are frequently encountered.

\section{References}

Amato, F., Cosentino, C., \& Merola, A. (2010). Sufficient conditions for finitetime stability and stabilization of nonlinear quadratic systems. IEEE Transactions on Automatic Control, 55(2), 430-434.

Boyd, S., Ghaoui, L. E., Feron, E., \& Balakrishnan, V. (1994). Linear Matrix Inequalities in System and Control Theory. SIAM Studies in Applied Mathematics, Philadelphia, USA.

Chen, A., \& Wang, J. (2012). Delay-dependent $L_{2}-L_{\infty}$ control of linear systems with multiple time-varying state and input delays. Nonlinear Analysis: Real World Applications, 13(1), 486-496.

Dhawan, A., \& Kar, H. (2011). An improved LMI-based criterion for the design of optimal guaranteed cost controller for 2-D discrete uncertain systems. Signal Processing, 91(4), 1032-1035.

Du, H., Zhang, N., \& Naghdy, F. (2011). Actuator saturation control of uncertain structures with input time delay. Journal of Sound and Vibration, 330(18-19), 4399-4412.

Huang, L., \& Mao, X. (2009). Robust delayed-state-feedback stabilization of uncertain stochastic systems. Automatica, 45(5), 1332-1339.

Liu, A., Yu, L., \& Zhang, W. A. (2011). $H_{\infty}$ control for network-based systems with time-varying delay and packet disordering. Journal of the Franklin Institute, 348(5), 917-932.

Oishi, Y., \& Fujioka, H. (2010). Stability and stabilization of aperiodic sampled-data control systems using robust linear matrix inequalities. $\mathrm{Au}$ tomatica, 46(8), 1327-1333.

Rubió-Massegú, J., Palacios-Quiñonero, F., \& Rossell, J. M. (2012). Decentralized static output-feedback $H_{\infty}$ controller design for buildings under seismic excitation. Earthquake Engineering and Structural Dynamics, 41(7), 11991205 .

Wang, C., \& Shen, Y. (2011). Delay-dependent non-fragile robust stabilization and $H_{\infty}$ control of uncertain stochastic systems with time-varying delay and nonlinearity. Journal of the Franklin Institute, 348(8), 2174-2190.

Wang, Z., Liu, Y., Wei, G., \& Liu, X. (2010). A note on control of a class of discrete-time stochastic systems with distributed delays and nonlinear disturbances. Automatica, 46(3), 543-548.

Zečević, A. I., \& Šiljak, D. D. (2004). Design of robust static output feedback for large-scale systems. IEEE Transactions on Automatic Control, 49(11), 2040-2044.

Zečević, A. I., \& Šiljak, D. D. (2008). Control design with arbitrary information structure constraints. Automatica, 44(10), 2642-2647.

Zečević, A. I., \& Šiljak, D. D. (2010). Control of Complex Systems: Structural Constraints and Uncertainty. Springer. 